\section{CEREBRO-SPINAL MENINGITIS, WITH REPORT OF CASES.}

BY J. F. SHELLEY, M.D.

RLMDALE, KAN.

The disease has been demonstrated bacteriologically to appear in epidemic and sporadic form. In sporadic cases it is difficult and frequently impossible, if severe and rapidly fatal, to differentiate this form of meningitis from that secondary to other infectious diseases, except by lumbar puncture, and therefore it is as yet impossible to suy how extensively the disease exists in sporadic form.

In 1880 Eberth supposed he had found in the micrococcus lanceolatus the essential cause of the disease, and since then until about 1895 it was generally accepted as the specific cause, the demonstration of a distinct micro-organism in 1887 by Weichselbaum, which he called the "diplococcus intracellularis meningitidis" notwithstanding. It seems that the organism found by Weichselbaum was supposed to be the lanceolatus (Flexner and Barker, Am. $J$. Med. Sciences, Vol. 107, p. 159), and it was not until Jaeger, Heubner and Finkelstein about nine years later took up the matter and confirmed the demonstrations of Weichselbaum that the meningococcus of Weichselbaum received general recognition. These investigators clearly differentiated between the two organisms both microscopically and by inoculation experiment. They found the lanceolatus far more virulent than the intracellularis.

Recently the epidemic in Boston through the researches of W. T. Councilman, Mallory and Wright (Amr. Jour. Med. Sciences, March, 1898), has thrown much light on the etiology of the disease, and has given us a clear definition of all the aspects of the micrococcus both in the organism and in culture. They made lumbar puncture in fifty-five cases and demonstrated the diplococci in thirty-eight of them. They think the negative results in the balance of the cases were not always because they were not present, and some of the punctures were made presumably after they had disappeared from the fluid.

In general he confirmed the results of Weichselbaum and Jaeger, but he thinks the capsule referred to by Jaeger as occasionally met with is simply a swollen condition in which the organism would not take the stain, except a small portion of the center. Councilman also confirms the statement of Weichselbaum that the diplococcus is decolorized by the Gram method, while Jaeger said coverslips could be stained with the Gram method. There seems to be some difficulty to differentiate between gonococci and the diplococcus intracellularis, but the latter vary more than the former in size and the meningococci are said to grow well on glycerin-agar.

The mode of entry and the avenue are still matters of very uncertain speculation. The nose, the ear and the intestinal canal all have been supposed by various observers to be the infection atrium. Strümpell believed there was a relation between coryza and epidemic meningitis, and if this be true, with the fact that Sternberg found the micrococcus lanceolatus in the saliva of 50 per cent. of healthy individuals, it was not hard to believe so long as this organism was supposed to be the cause of the disease, that the nares served a very probable way of infection. That the real microbe of the disease is not so constantly found in these parta does not, however, bar the possibility of thus infecting the meninges. Weigert made note of purulent inflammations of the nasal sinuses accompanying the disease. The writer has also seen a case (noted below) in which there was a copious discharge of purulent matter from the nose. But Flexner and Barker in their autopsies of acute cases at Lonaconing found the nasal sinuses free from disease. They believed the alimentary tract to be the source of infection. But it may be noted that they at that time still attributed the disease to the micrococcus lanceolatus.

The source of the infection outside of the body is likewise a matter shrouded in obscurity. We know that it is likely to manifest itself in overcrowded, illkept quarters. That it is most frequent during winter and spring, and that sudden lowering of temperature especially in wet weather is generally during an epidemic accompanied or followed immediately by the appearance of new cases. These are questions that will probably be explained in the future, but at present they are only suggestive.

There is as yet no evidence to prove the disease contagious. Inoculation experiments since the isolation of the diplococcus intracellularis and its distinction from the pneumococcus have been mainly negative, except when introduced into the pleura or the meninges. If inoculated into the latter a typical meningitis is the result and in the pleura it causes a pleuritis.

The disease is distinctive in that it is essentially primary, while other forms of meningitis are generally secondary to disease located elsewhere, or they are caused by injury in which the seat of infection is made to communicate with external parts. Councilman says, " in tuberculous meningitis we have never found a single case in which the lesion in the meninges could be regarded as primary. The only two cases of apparently primary infection were in the two pneumococcus cases noted, and in one of these the infection may have come from the intestinal canal."

The symptoms are varied in extent and intensity. The onset is usually abrupt and agonizing. Without premonition, the patient is seized with violent pains in the head and back, chill, fever and vomiting. In the severer cases these symptoms, with extreme restlessness, merge into active delirium and spasm, and death may take place in a few hours or days.

The headache is a prominent symptom throughout the course of the disease. It may be general or localized, but is apt to be most marked in the frontal and occipital regions. It may be continuous with acute exacerbations, or it may remit only to assert itself at intervals of various length. It sometimes recurs with striking regularity, but at other times there is no regularity whatever to the returns. Light to the eyes increases the pain in the head, and pains the eyes. The pain in the back is equally intense, and has the same characteristics. It soon extends to the spinal nerve-roots and to the nerves themselves, and motion becomes very painful. Hyperesthesia is soon marked in localized places or it may be general. Some authors mention anesthesia. I have not noted this symptom but can easily see why the exudate should produce it. The pain of meningitis is apt to be of a sharp lancinating character.

Temperature is generally moderate and bears no special relation to the severity of the disease. It may 
be subnormal, or in approaching dissolution may reach a very high degree. There is no regular course of fever in meningitis as in typhoid.

Usually vomiting does not continue beyond twentyfour hours, but may be a prominent symptom throughout the course of the disease. More often it is recurrent. The cause of the early vomiting is central, but later, when the secretions are much disturbed, it may be partially due to indigestion. The tongue, slightly coated in mild cases, soon becomes heavily covered with a dark to brown coat, and presents a dry and cracked appearance in the so-called typhoid cases. The mouth is not infrequently covered with sordes. A characteristic feature of the disease is the retracted abdomen, but this condition may be changed in those cases of severe grade with prominent digestive symptoms, and in which intestinal secretions are greatly modified. It is not uncommon to find the abdomen tender, but this is due to the hyperesthesia general over the body, and must not be mistaken for the tenderness of ulcerated intestinal glands. The early history, the absence of the typhoid eruption, want of gurgling in the iliac fossa, and tenderness extending over the entire abdomen, rather than its localization in the iliac region, will usually serve to differentiate it from typhoid.

The pulse is variable and may be very rapid and feeble or it may be fairly hard and slow. It is frequently intermittent, dicrotic and irregular. It may be very rapid at first and then become abnormally slow, or the reverse may be true. It often changes in the same case and bears no relation to the temperature range.

Respiration is usually slightly increased in frequency in cases not complicated with pneumonia, and when so complicated, or in congestion of the lungs, it is increased in proportion to the lung affection. It may be Cheyne-Stokes in character, more especially in cases complicated with albuminuria. Involvement of the nerves of respiration may cause the most painful dyspnea. This may also be caused by disturbed innervation of the larynx. Sighing respiration is also noted.

The most constant form of eruption is the herpetic, and appears about the face and neck. The lips are mostly affected. A petechial eruption on the body or limbs is not quite so common, but is perhaps more characteristic of the disease. Other changes are noted in the skin, at times. The eruption bears no relation to the severity of the other symptoms and it may be absent in the severest cases.

The urine varies from scanty secretion with high specific gravity to polyuria with specific gravity 1002 to 1005. It is frequently alkaline and deposits excess of phosphates. The triple phosphates are especially abundant. Albuminuria is not an infrequent complication and should be constantly looked for. One of the author's cases had vesical paralysis; also paralysis of the sphincter ani.

The bowels are constipated, with very few exceptions. There are, perhaps, more exceptions among children than adults. Flexner and Barker report four cases in the Lonaconing epidemic, which were complicated with dysentery, and which they made the subject of special study. The discharges contained mucus, pus and blood, and the predominating organism was a lancet-shaped coccus, in pairs, apparently encapsulated. They also found bacilli resembling the coli communis, and short chains of streptococci. One of the bacillus. author's lighter cases was dysenteric, with mucous pus and blood presenting.

Motor and sensory disturbances are a general part of the disease. Vision is disturbed in many cases, and metastatic optic neuritis is not uncommon. Strabismus and nystagmus are common. Sense of taste and smell may be perverted and hearing lost. The latter may, however, be due to suppurative inflammation of the middle ear, as well as to sensory disturbances. Muscular contraction soon manifests itself and we have the retracted head, opisthotonos, orthotonos (Osler), the arms flexed on the chest, the thighs flexed on the abdomen and the legs on the thighs. In this condition the case presents a typical picture.

Flexner and Barker in their report, and Osler, in "Nervous Diseases," edited by Dercum, cite a symptom described by Kernig as pathognomonic of the disease. It is what they call flexion-contracture (called by Kernig, beuge-kontraktur), by which is meant an inability to extend the leg while the thigh was flexed on the abdomen, or at right angles to it, this inability to extend the leg disappearing as the thigh is extended. I am surprised that, in consideration of the hyperemic and inflammatory condition of the nerves and nerveroots and consequent hyperesthesia, it did not occur to them that this was due to tension on the sciatic nerve and that the same condition obtains in other painful affections of this nerve, as in sciatica neuritis. However, it may serve an important office in differentiating joint complications of the disease from nerve pain, as it differentiates hip-joint disease from sciatica. Epistaxis is a common symptom.

Typical cases not of the severest type are easily recognized after a few days. On the other hand, great difficulty may be experienced very early in the disease, and especially in severe sporadic cases. Malignant scarlatina, in which death occurs before the eruption appears, can be distinguished only by the history of the contagion. The writer has had two such cases, one of which gave no history of contagion, but three others in the same family following immediately with scarlatina, gave evidence for the diagnosis. The other was a child of 3 years in a family in which three older brothers had scarlatina and were convalescing. Without premonition it was suddenly attacked with vomiting, and this was followed immediately by convulsions, the eyes set, teeth tightly closed (trismus), the head drawn back, the back in opisthotonos and frothing at the mouth; soon becoming comatose, it died in about six hours after the first symptoms. Involvement of the nerve centers in the course of other infectious diseases, as diphtheria, pneumonia, typhoid, etc., can usually be differentiated by the symptoms of the primary diaease. We think there is no longer any doubt that those cases of the acute infections which manifest the central symptoms are cases of involvement of the nerve centers proper. When we say central symptoms, we do not mean the ordinary delirium of typhoid, nor the coma of uremia. Postmortems in typhoid with central symptoms have revealed the specific typhoid bacillus in the meninges (Ohlmacher, JoURNaL American Medical Association, Vol. 29, p. 419), likewise the micrococcus lanceolatus in meningitis complicating pneumonia. Councilman found, besides the diplococcus intracellularis, the tubercle bacillus, pneumococcus, streptococcus, bacillus pyocyanus, staphylococcus pyogenes aureus and the anthrax 
Tuberculous meningitis is recognized by its insid. ious and gradual development.

Where two or more members of a family are simul. taneously attacked, as in the recent cases of the writer, it is easily mistaken for acute poisoning.

In differentiating meningitis, the fact that a case recovers may have an important significance. Councilman says, "We believe that all infections of the meninges, other than the diplococcus intracellularis, are fatal." The one most important means of differentiating, however, is the examination of the exudate obtained by lumbar puncture, both with the microscope and in culture experiment. Lumbar puncture is said to be made not only with impunity, but in some cases with actual benefit to the patient (Councilman).

Medical treatment, for the main part, aims at the comfort of the patient and the relief of complications as they arise. He should be placed in a large, well ventilated room and the light modified to his comfort. The diet should be liberal and nutritious, milk in quantity being best suited to most cases. Opium in some form is indicated. It relieves pain, quiets the nervous system, lowers the pulse and increases its volume, and frequently lowers the temperature, thus making it possible to better nourish the patient. Bromids are important adjuvants to morphin or other opiates. An important matter in the administration of opiates is to give them cautiously, but increase until the desired effect is produced. They increase the desire for food. To move the bowels, I prefer calomel in small and divided doses, generally 0.065 gram in quarters or sixths; this may be given, when necessary, throughout the course of the disease. These fractions are given every fifteen minutes until the desired quantity is administered.

It is not intended to give here a complete résumé of treatment, since there is nothing new, and it is well discussed in the various text-books, as Hare's "System of Practical Therapeutics," Loomis' "Practice," Dercum's "Nervous Diseases," Rotch's "Pediatrics," and others.

Though the patient becomes very sensitive to touch, at times gentle sponging is grateful, and pain is often markedly modified by gently stroking the part with a soft hand. The surface of the body must be kept warm with flannel clothing, and we believe with Bartholow, that cold to the head is not the best treatment. Small blisters to the back of the neck are useful.

The following cases are of special interest for the simultaneous onset, in three of them, and the severity of two, with complete recovery in forty and fortythree days respectively.

The family of $\mathrm{H}$. lived in a dilapidated house of four small rooms during the winter of 1897 and 1898 . It consisted of ten members and the house was poorly furnished, there being but three beds and those very scantily equipped. In fact, there was but one bed that was in a condition for occupancy. The food was probably not all that it should have been; in fact, at the onset of the disease, the family became a county charge and it was found necessary to supply linen, while the balance of the family, except just enough to care for the sick, were removed to other quarters. Feb. 23, 1898, three members of the family, the father, aged 54, and two boys, aged 8 and 10, were simultaneously attacked with headache, pain in the back, chill, fever, vomiting and restlessness. The oldest boy suffered least and his proved an abortive case.

After twenty-four hours his symptoms abated and in a week he was practically. well, though the petechial eruption, especially on his legs, was far more marked than in the other cases, and the herpetic eruption was not inconsiderable. Both the others became delirious, the delirium being of a noisy character, especially in the man. He was, for a number of days, constantly up and down, often laboring under the delusion that he was ordered either to get up or return to bed, and no assurance to the contrary would induce him to remain in bed. Delusions and hallucinations were a prominent feature of his delirium. Cards, pictures and other wall hangings had to be removed, as they presented to him an army of menacing figures that gave him no slight concern. His speech was not noticeably incoherent, and he would have delusive mental impressions of such lasting effect that he would narrate them as facts, time and again, for days. Delirium did not last so long in the 8 year old boy, though most of the other symptoms were more marked. The herpetic eruption about the mouth was most marked in the man, vasicles forming and rupturing and the lesions coalescing, it presented one large crusted sore. It prevented closure of the mouth and increased the tendency to changes. Petechial spots were noted on the limbs and a light mottling could be seen over the entire body. There was a painful stiffness of the body and limbs, most marked in the right leg, in the knee of which he said he had rheumatism and wanted a plaster put on it. Examination revealed no swelling or redness, and it could be flexed and extended without pain, when the thigh was not flexed at the hip (Kernig's sign). The head was retracted for about two weeks, and for about twice that time he was unable to lower the chin. There was no noticeable opisthotonos. Pain in the back, head and right leg, at first constant, after several weeks became remittent, and lasted throughout the course of the disease. There were photophobia and deafness. The teeth were covered with sordes, and the tongue had a typhoid appearance. About the beginning of the second week there was a sudden discharge of purulent matter, in large quantity, from the nose. It continued for several days. There was general hyperesthesia and movements were generally painful. The pulse ranged from 85 to 110 . It was soft and unsteady and stimulants were given, in moderation, after the first or second week. He was a habitual user of intoxicants and in considerable quantity, though he seldom became intoxicated. Tem. perature ranged from 99 to 102 degrees F., but was at times subnormal. Respiration was not materially modified. During the second week there was albuminuria, which lasted about a week. Potassii acetas was used, with spiritus etheris nitrosi to relieve this complication. During the third week there was vesical paralysis, requiring catheterization. Constipation existed throughout the disease and was relieved with calomel, as indicated. Milk was the main diet, of which he probably took, on an average, a quart a day, and never less than that half that quantity, except during the first fow days.

The 8 year old boy had less eruption than either of the others. The herpetic spots were isolated and few in number, although he had some about the mouth, nose, and one on the upper lid of the right eye. His pains were excruciating, except as they were modified by large doses of morphin. There was no region of the body in which pain was not complained of. 
Hyperesthesia was marked throughout. Retraction of but was soon discontinued, as it seemed to increase the head became so marked that deglutition became at hyperesthesia and general symptoms of neuritis. We times very difficult or impossible. There was extreme think this remedy, if given at all, should be given opisthotonos, which, with the retraction of the head, cautiously and not so long as symptoms of neuritis continued for fully five weeks. His arms were flexed are at all marked.

on the abdomen or chest, the lower limbs drawn up and flexed at the knees. The Kernig symptom was present for some time, but later any attempt at extension became painful. He lay on his side in the manner indicated and, to take nourishment, with some assistance, he would turn on his abdomen, which would throw the retracted head in an upright position so that he could receive it in a fairly natural manner. In assuming this position, however, he never failed to implore his attendant to be careful.
There was deafness and intolerance of light. His pulse during the first week ranged from 100 to 120 per minute, but during the second week it dropped to from 55 to 70 (mostly beating about 60 ) where it remained for some weeks, then it again averaged about 90 , and so remained until he was discharged. Temperature for first thirty-six hours was from 100 to 103. After that seldom above 101, and ranged mostly from 99 to 100 . At times it was subnormal. Respiration was from 20 to 30 , mostly about 24 per minute. During the second, third and fourth weeks it was markedly Cheyne-Stokes in character, the period of apnea lasting as long as twenty-five seconds. The pulse was also much influenced in its rhy thm and volume during these changes in the respiration. The Cheyne-Stokes phenomenon was most marked during a period of albuminuria lasting several days, during the fourth week. Twice he had an attack of dyspnea with violent, painful constriction in the chest. His face had the anxious expression of one suffocating, while he tore wildily at the clothes about the chest. The urine was examined in both cases every day or two, both chemically and microscopically. The boy's urine was frequently abundant, with low specific gravity, $100 \tilde{s}$ alkaline, with excess of triple phosphates. At times, however, it was scanty, with high specific gravity, 1025. The father's urine was generally fairly concentrated.

Both these cases received morphin and the boy took, during his illness, very much more than his father. At times the boy required 0.016 gram every four hours to control the pain. It was, however, given mostly in 0.008 gram duses every two hours, but while giving it this way an extra dose was required at times. The morphin was only given as required, and as he began to improve intervals of half a day to a day would elapse in which it was not necessary to give morphin, but when the pain did recur it would yield to nothing short of the full dose. Even the very last paroxysm of pain required no less than three doses of the morphin. Atropia was always given with the morphin but not increased with it. Small doses of bromid were given throughout the illness. With the large doses of morphin required by the boy, his desire for nourishment did not seem impaired, and it is worthy of note that he took an average of a third more milk than his father, taking seldom less than a quart per day. With a view of promoting gastric digestion dilute hydrochloric acid was given in small doses. It did not, however, improve the digestion, but seemed to aggravate the general symptoms. The potassii acetas, on the other hand, seemed to have a favorable influence. Fowler's solution, in small doses, was tried as a reconstructive after convalescence was established,

February 25 another boy, aged 15 , became sick with symptoms of the disease, but they were mild, and in a week he was again feeling quite well. Two other members of the family, some days later, became sick and were indisposed for several days, but the symp. toms did not point sufficiently to the disease to include them. There was, however, one other case in another family, a little girl 8 years old, who was sick fourteen days. Early symptoms were the same as those of cases described. There was, however, in this case, marked dysenteric discharges containing mucus, pus and blood. The case was also markedly intermittent in type. The pain was controlled with acetanilid, no opiate being given. Bromid was given in small quantity.

All these cases were frequently and carefully examined for signs of pulmonary changes, but with negative results, at least so far as physical signs were concerned. The disturbed or slightly increased respiration at times noted was due either to disturbed central innervation or peripheral nerve involvement making normal respiration painful, and hence the more frequent but shallow respirations.

\section{OBSERVATIONS UPON THE SPECIFIC TREATMENT OF TUBERCULOSIS.}

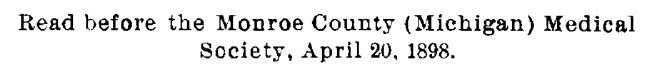

Briefly, today the pathology of tuberculosis takes into consideration but one real causative factor, the bacillus. Yet apart from this assumption I can not disabuse my mind of the impression that we may and do have a form of phthisis in which this form of bacillus does not directly figure. Such cases are characterized by a low form of inflammatory action, a fibrosis, if you please, with little or no fever. The expectoration is mucopurulent and profuse; consolidation, usually at the base, is present, with the usual. classic symptoms. I have made many examinations of the sputum of these cases, finding no bacilli. I take it that this class of cases constitutes the longdrawn-out ones, lasting perhaps for years. They are doubtless susceptible to true tubercular infection, which infection usually runs to a rapid termination. The comparative absence of fever and the failure to find the bacilli, point quite conclusively to this form of consumption. If, then, we have among us this separate type of disease, we perhaps have an explanation of some failures along the lines of specific treat. ment.

Since the discovery of the bacilli of tuberculosis by Koch, in 1882, there has been a renewed impulse along various lines of investigation, with a view to find some means or remedy inimical to the growth of this bacillus.

If we stop to consider the fearful mortality of consumption we shall see how eminently humane and just are the efforts to mitigate, cure or control this terrible scourge. The true physician will strive to aid in this conquest, will try all things and hold fast to that which is good. In the use-of Koch's crude 